\title{
Epilepsy duration and seizure outcome in epilepsy surgery
}

\section{A systematic review and meta-analysis}

Johan Bjellvi, MD, Ingrid Olsson, MD, PhD, Kristina Malmgren, MD, PhD,* and Karin Wilbe Ramsay, PhD*

Neurology ${ }^{\circledR}$ 2019;93:e159-e166. doi:10.1212/WNL.0000000000007753

\section{Abstract}

\section{Objective}

To conduct a systematic review and meta-analysis on the effect of earlier or later resective epilepsy surgery on seizure outcome.

\section{Methods}

We searched the electronic databases PubMed, EMBASE, and Cochrane Library for studies investigating the association of epilepsy duration and seizure freedom after resective surgery. Two reviewers independently screened citations for eligibility and assessed relevant studies for risk of bias. We combined data in meta-analyses using a random effects model. We assessed the certainty of evidence according to Grading of Recommendations Assessment, Development and Evaluation (GRADE).

\section{Results}

Twenty-five studies were included, 12 of which had data suitable for meta-analyses. Comparing seizure outcome if epilepsy surgery was performed before vs after 2, 5, 10, and 20 years of epilepsy duration, and comparing epilepsy duration $<5$ years to $>10$ years, we found significant effects favoring shorter duration with risk differences ranging from 0.15 to 0.21 and risk ratios ranging from 1.20 to 1.33 ( $p<0.01$ for all comparisons). According to GRADE, we found low certainty of evidence favoring shorter epilepsy duration before surgery.

\section{Conclusion}

People with shorter epilepsy duration are more likely to be seizure-free at follow-up. Furthermore, there is a positive association between shorter duration and seizure freedom also for very long epilepsy durations. Patients who might benefit from epilepsy surgery should therefore be referred for presurgical assessments without further delay, regardless of epilepsy duration. The low certainty of evidence acknowledges concerns regarding study heterogeneity and possible residual confounding.

\author{
Correspondence \\ Dr. Bjellvi \\ johan.bjellvi@vgregion.se
}




\section{Glossary}

GRADE = Grading of Recommendations Assessment, Development, and Evaluation; ILAE = International League Against Epilepsy; $\mathbf{R C T}=$ randomized controlled trial; $\mathbf{R D}=$ risk difference; $\mathbf{R R}=$ risk ratio; $\mathbf{S B U}=$ Swedish Agency for Health Technology Assessment and Assessment of Social Services.

Epilepsy surgery is an evidence-based treatment option for people with drug-resistant epilepsy. ${ }^{1-3}$ Drug resistance is defined by the Task Force of the International League Against Epilepsy (ILAE) Commission on Therapeutic Strategies as failure of adequate trials of 2 tolerated, appropriately chosen and used antiepileptic drug schedules to achieve sustained seizure freedom. ${ }^{4}$ This definition facilitates for nonspecialists to recognize patients with drugresistant epilepsy and refer them promptly to specialist centers for evaluation of epilepsy surgery or other specialized treatments.

Despite this, referral patterns have not undergone any major changes over the last decades. ${ }^{1,2,5,6}$ Although the importance of early referral has been emphasized repeatedly, ${ }^{7}$ epilepsy surgery is still considered by many neurologists to be the last resort. ${ }^{8}$ Many patients who are offered surgery have had drug-resistant epilepsy for half of their lives. ${ }^{9}$ A number of observational studies have suggested that a short duration of epilepsy is associated with better seizure outcome after resective epilepsy surgery. If corroborated, this finding further underscores the importance of early referral. However, the study designs vary, the cohorts are often limited, and varying resection types have been studied.

The aim of this study was therefore to assess the evidence comparing the effect of earlier or later resective epilepsy surgery on seizure outcome after surgery in a systematic review and meta-analysis.

\section{Methods}

\section{Inclusion criteria}

The review was part of a larger project investigating the effect, safety, cost-effectiveness, and ethical aspects of several methods for treatment and diagnosis of epilepsy, conducted at the Swedish Agency for Health Technology Assessment and Assessment of Social Services (SBU), and initiated in collaboration with the National Board of Health and Welfare. Main selection criteria and methods of analysis for the review were specified and documented in advance. The systematic review was conducted in accordance with the PRISMA statement, ${ }^{10}$ following an a priori but unpublished protocol available on request.

The criteria for eligibility were the following:

- Population. Individuals with drug-resistant epilepsy at all ages, study population $\geq 30$.
- Intervention and comparison. Resective epilepsy surgery performed at different time intervals after epilepsy onset. - Outcomes and measures. (1) Postsurgical seizure outcome expressed as proportions of patients with seizure freedom grouped by various presurgical epilepsy durations or regression analysis or other measure of association between presurgical epilepsy duration and postsurgical seizure freedom (reported as odds ratio, risk ratio, hazard ratio); (2) surgery-related complications related to presurgical epilepsy duration. Furthermore, seizure freedom should be reported as ILAE Class I (seizure-free without aura), ILAE Class I + II (seizure-free with or without aura), ${ }^{11}$ Engel Class Ia (seizure-free), or Engel Class I (no disabling seizures). ${ }^{12}$ Seizure freedom should be assessed at the earliest 12 months after surgery (an exception was made if a small minority of the study population was assessed earlier). Studies that only reported mean or median duration of epilepsy for patients grouped by seizure outcome were not included.

- Study design. Randomized controlled trials (RCT) or observational studies with prospective or retrospective design.

- Language limitations. English, Swedish, Norwegian, or Danish language.

- Publication type. Publications in peer-reviewed journals published in 2000 or later.

\section{Search strategy}

We searched the literature in Cochrane Controlled Register of Trials (Central), PubMed (NLM), and EMBASE (Elsevier) on 2 occasions, covering literature published from 2000 to November 10, 2017. The search terms "epilepsy" and "surgery" and related terms were used. The detailed search strategy is available in appendix e-1 (doi.org/10.5061/dryad. g8c0vm8). We examined the reference lists of included studies for additional relevant studies.

\section{Study screening and selection}

Two reviewers screened the titles and abstracts independently. Full-text articles were retrieved if one or both reviewers considered a study potentially eligible. At least 2 reviewers read the full texts, and any disagreement regarding eligibility was resolved by discussion. Only unique nonoverlapping study populations were included.

Two reviewers independently assessed eligible studies for risk of bias using a standardized tool developed at SBU for observational studies (sbu.se/en/method/). We scored studies as having high, medium, or low risk of bias, based on the following domains: selection bias (including assessment of 
confounders), performance bias, detection bias, attrition bias, reporting bias, and potential conflicts of interest. Only studies with low or medium risk of bias were included.

\section{Standard protocol approvals, registrations, and patient consents}

No additional ethical approval was required for this systematic review and meta-analysis.

\section{Data extraction}

For all included studies, we extracted country, patient characteristics such as age, sex, and type of epilepsy, type of surgery, method of data collection, definition of seizure freedom, length of follow-up after surgery, and outcome. Proportions of patients with seizure freedom at different epilepsy durations expressed as $\mathrm{n} / \mathrm{N}$ were extracted or recalculated from percentages when possible. The full extracted study data are available in appendix e-2 (doi.org/10.5061/dryad.g8c0vm8).

\section{Synthesis and statistical analysis}

Meta-analyses were performed using Review Manager (RevMan) version 5.3 (Copenhagen: The Nordic Cochrane Centre, The Cochrane Collaboration, 2014), employing the Mantel-Haenszel method for binary outcomes. We used a random effects model for all analyses, as clinical heterogeneity was present in the included studies. Outcomes were expressed as risk difference (RD) and risk ratio (RR) with $95 \%$ confidence intervals. We considered $p$ values $<0.05$ as significant. The degree of statistical heterogeneity was assessed using the $I^{2}$ index.

\section{Assessment of evidence}

The certainty of evidence was assessed according to Grading of Recommendations Assessment, Development, and Evaluation (GRADE), where evidence levels are expressed as strong, moderate, low, or insufficient. ${ }^{13}$ Preliminary certainty of the evidence is classified as high if the results are based on data from RCT, otherwise as low. Each outcome is assessed separately and can be rated down from the preliminary level by the 5 risk domains in GRADE: overall risk of bias across studies, inconsistency, indirectness, imprecision, and publication bias. Observational studies can be upgraded from the initial level based on large magnitude of effect, presence of dose-response gradient, or presence of opposing confounding effects.

\section{Data availability}

Data are available to qualified investigators on request to the corresponding author.

\section{Results}

\section{Search results and study selection}

The electronic database search strategy yielded 8,074 citations, from which 115 articles were obtained and examined in full text. Of these, 25 studies fulfilled the eligibility criteria (figure 1). ${ }^{6,14-37}$ All the included studies were observational
Figure 1 PRISMA flow diagram for the literature search and study selection

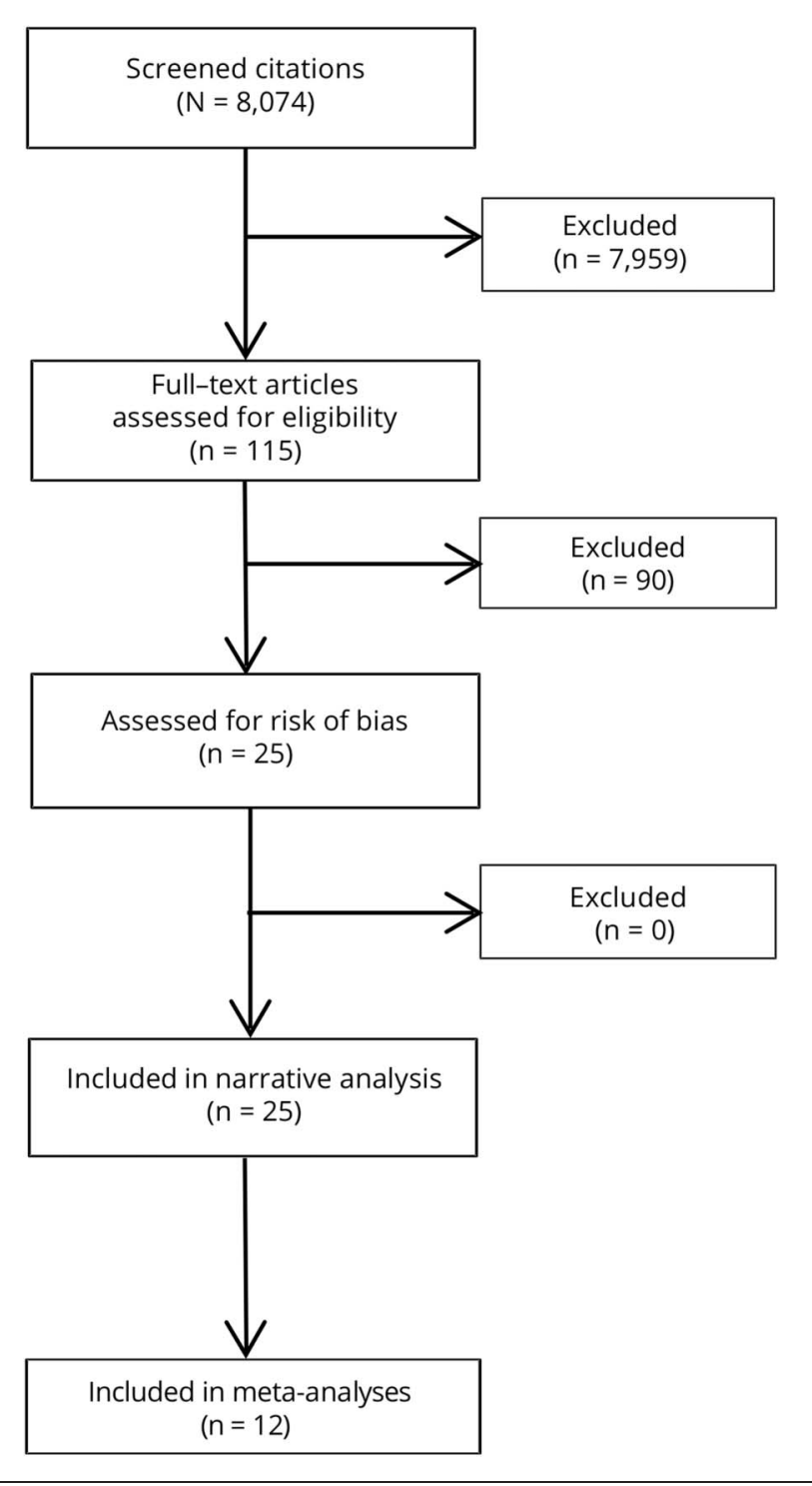

studies with varying study design. As expected, we found no RCT that investigated the targeted question. We found no study that reported on association between presurgical epilepsy duration and surgery-related complications.

\section{Characteristics of included studies}

In total, the studies included data from 3,746 patients with drug-resistant epilepsy who had undergone resective surgery. For details, we refer to the extracted study data (appendix e-2, doi.org/10.5061/dryad.g8c0vm8). Most studies were retrospective analyses of patient data from national or local databases or medical records. Some studies included only temporal lobe resections, ${ }^{14,17,21-23,26,27,33}$ frontal lobe resections, ${ }^{32}$ posterior resections, ${ }^{16,24,31}$ or all extratemporal resections, ${ }^{19}$ while other studies included resections regardless of the location. Several cohorts comprised only epileptogenic lesions. ${ }^{6,17,21,25,29,30}$ Age range, sex ratio, study period, and follow-up time varied across the studies. 
Most studies included both children and adults, while 4 cohorts comprised only children and adolescents up to the age of 18 years, ${ }^{17,31,32,34}$ and 5 only adults. ${ }^{14,19,22,23,36}$ Several studies included patients under 5 years of age. ${ }^{17,18,20,21,24,30-32,34,35}$ All studies reported seizure freedom (including or excluding aura) in accordance with the eligibility criteria. Various measurements for associations of presurgical epilepsy duration and postsurgical seizure freedom were reported in the studies, such as proportions, odds ratio, and hazard ratio.

\section{Risk of bias assessment}

All eligible studies were classified as having an overall moderate risk of bias; hence, no study was excluded due to high risk of bias. This was partly a consequence of the criteria decided upon at the eligibility state as regards reporting of outcome and length of follow-up. Known predictors for seizure outcome, such as MRI findings, histopathology, and preoperative seizure frequencies, were not reported in relation to epilepsy duration. We recognized that confounding effects could be present and that the direction of these potential biases (favors short duration or favors long duration) was difficult to predict.

\section{Synthesis of outcomes}

We used 2 approaches to evaluate the assembled effect from the included studies. First, a narrative synthesis was undertaken, where we examined all the included studies for the reported effect on association between epilepsy duration and postsurgical seizure outcome. According to the narrative examination, most of the studies reported associations in favor of shorter duration ${ }^{6,15-19,21-35}$; in some cases, these results were statistically significant. A few studies reported results that suggested no association (e.g., odds ratio close to $1),{ }^{14,20,36}$ and one study reported on association in favor of longer duration. ${ }^{37}$

Second, we used a meta-analysis approach, where data from studies reporting proportions of seizure freedom before and after a certain time of presurgical epilepsy duration were assembled in meta-analyses. The breakpoints (before vs after 2, 5,10 , and 20 years of epilepsy duration, respectively) were chosen so that the most frequently reported data were used. Twelve studies $(\mathrm{n}=1,545)$ reported data on this format. $^{6,15,22,24,27,28,30,31,33-35,37}$

The results from the meta-analyses are summarized in figure 2. Three studies $(n=288)$ reported data on seizure freedom at epilepsy duration $<2$ years compared to $>2$ years, ${ }^{6,31,34} 4$ studies $(n=551)$ reported seizure freedom at epilepsy duration $<5$ years compared to $>5$ years, ${ }^{6,24,30,34} 10$ studies ( $\mathrm{n}=$ $1,376)$ reported data on $<10$ years compared to $>10$ years. ${ }^{6,15,22,24,27,30,33-35,37}$ Three studies $(\mathrm{n}=346)$ reported data on $<20$ years compared to $>20$ years. ${ }^{6,22,28}$ Finally, we compared epilepsy duration $<5$ years to $>10$ years, in order to investigate if a larger time gap in epilepsy duration resulted in a larger effect. Data for this comparison were found in 4 studies $(n=430) .{ }^{6,24,30,34}$ All comparisons showed significant effects favoring shorter duration with $\mathrm{RD}$ ranging from 0.15 to 0.21 and RR ranging from 1.20 to 1.33 ( $p<0.01$ for all comparisons).

\section{Strength of evidence}

We assessed the strength of evidence for the general question of association between presurgical epilepsy duration and postsurgical seizure freedom. Thus, the specific comparisons targeted in the meta-analyses (before vs after 2, 5, 10, or 20 years of epilepsy duration) were not judged individually. The assessment was based both on the narrative analysis of all the included studies and on the meta-analyses.

The grading of evidence started at "low," as the material in this review consisted of observational studies. The estimate of association varies among the studies, but most of them suggest an association favoring shorter epilepsy duration. The meta-analyses support this assumption by showing significant effects favoring shorter epilepsy duration for all comparisons. We recognized that the individual studies had some weaknesses regarding risks of bias and that there were some concerns regarding inconsistency and indirectness. However, these factors did not justify downgrading the evidence level from the already low starting point. Nor were there reasons strong enough to upgrade the evidence level. Hence, this review provides low certainty of evidence for an association between shorter presurgical epilepsy duration and a higher proportion of patients with postsurgical seizure freedom (for summary of findings, see the table).

\section{Discussion}

The present study shows an association between shorter duration of epilepsy and better seizure outcome after resective epilepsy surgery in a systematic review and meta-analysis. The discussion about the importance of earlier epilepsy surgery has been around for a long time and motivated US researchers to initiate an ambitious RCT, ERSET, to determine whether surgery in patients with drug-resistant temporal lobe epilepsy soon after failure of 2 antiepileptic drug trials is superior to continued medical management. ${ }^{2}$ A sample size of 200 participants was originally planned, but the trial had to be terminated prematurely. Only 38 patients were recruited with a mean epilepsy duration of 10.9 years, ${ }^{2}$ which though it may seem rather long is much shorter than the mean epilepsy duration of 19.7 years in the first randomized controlled trial. ${ }^{1}$ Nevertheless, even in this limited cohort, resective surgery and antiepileptic drug treatment resulted in a lower probability of seizures during the second year of follow-up than continued drug treatment alone.

In the ERSET study, the authors comment that obtaining referrals from the community was difficult, although they do not discuss the reasons for this further. ${ }^{2}$ Among the reasons most often cited for not referring patients are that health care providers are negative to the treatment option of epilepsy surgery, ${ }^{38}$ 
Figure 2 Meta-analysis of seizure freedom with respect to different epilepsy durations

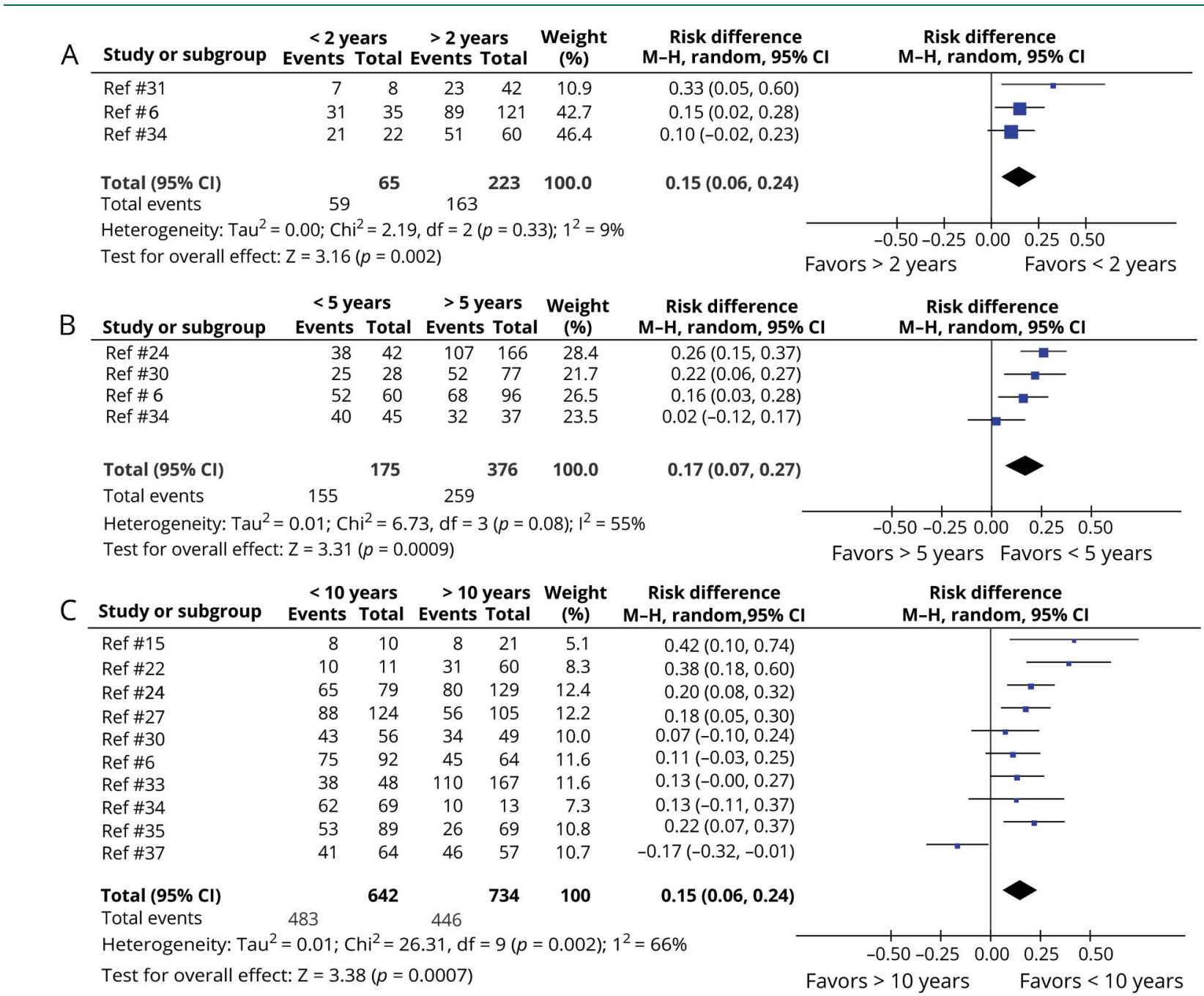

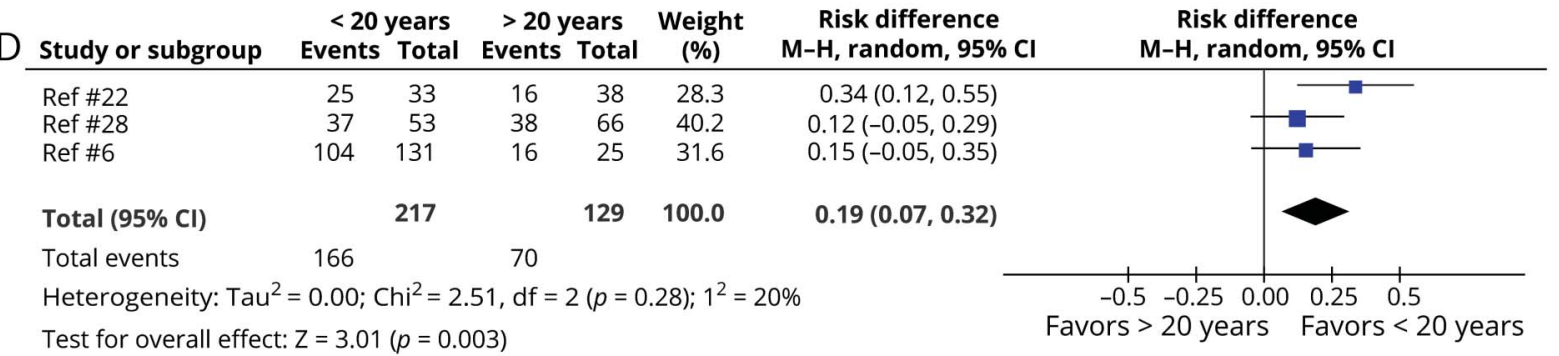

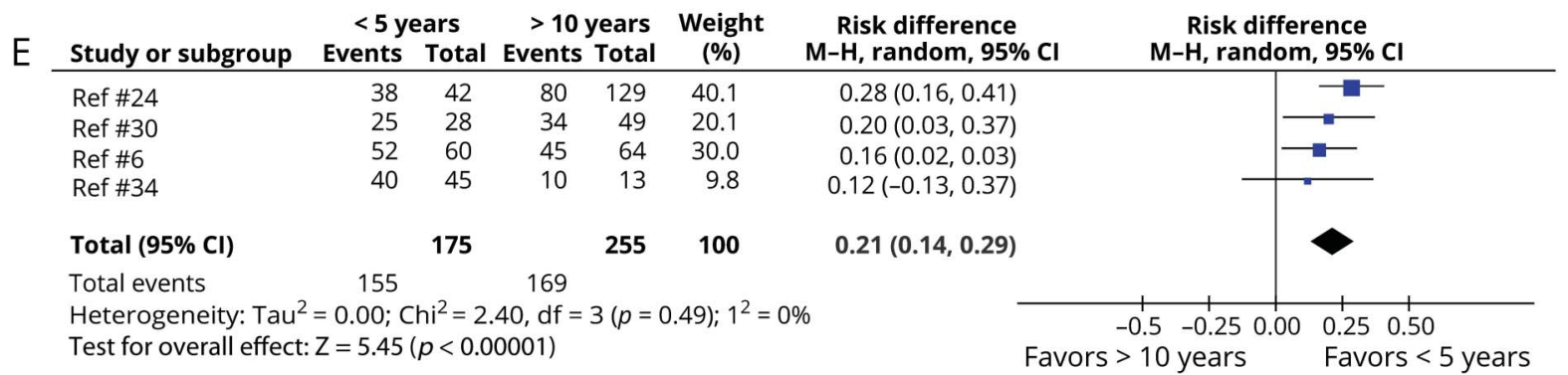

$\mathrm{Cl}=$ confidence interval; $\mathrm{M}-\mathrm{H}=$ Mantel-Haenszel.

or that they are not aware of which patients to refer. ${ }^{39}$ It is also understandable that patients may be hesitant early on to accept open brain surgery for their epilepsy, though several studies have illustrated that they tend to overestimate the risks. ${ }^{38,40-42}$ 
Table Findings for the association of epilepsy duration to surgery outcome

\begin{tabular}{|c|c|c|c|c|c|}
\hline Outcome & Comparison/analysis & $\begin{array}{l}\text { No. of participants } \\
\text { (studies) }\end{array}$ & $\begin{array}{l}\text { Absolute effect, RD } \\
(95 \% \mathrm{Cl})\end{array}$ & $\begin{array}{l}\text { Relative effect, RR } \\
(95 \% \mathrm{CI})\end{array}$ & $\begin{array}{l}\text { Strength of evidence } \\
\text { (GRADE) }\end{array}$ \\
\hline \multirow[t]{6}{*}{$\begin{array}{l}\text { Seizure } \\
\text { freedom }\end{array}$} & $\begin{array}{l}\text { Duration }<2 \text { years compared to } \\
>2 \text { years }\end{array}$ & $288(3)$ & $0.15(0.06 ; 0.24)$ & $1.20(1.05 ; 1.39)$ & Low certainty of evidence \\
\hline & $\begin{array}{l}\text { Duration }<5 \text { years compared to } \\
>5 \text { years }\end{array}$ & $551(4)$ & $0.17(0.07 ; 0.27)$ & $1.24(1.08 ; 1.42)$ & \multirow[t]{5}{*}{ Favors shorter duration } \\
\hline & $\begin{array}{l}\text { Duration }<10 \text { years compared } \\
\text { to }>10 \text { years }\end{array}$ & $1,376(10)^{a}$ & $0.15(0.05 ; 0.24)$ & $1.25(1.09 ; 1.43)$ & \\
\hline & $\begin{array}{l}\text { Duration }<20 \text { years compared } \\
\text { to }>20 \text { years }\end{array}$ & $346(3)$ & $0.19(0.07 ; 0.32)$ & $1.33(1.08 ; 1.65)$ & \\
\hline & $\begin{array}{l}\text { Duration }<5 \text { years compared to } \\
>10 \text { years }\end{array}$ & $430(4)$ & $0.21(0.14 ; 0.29)$ & $1.32(1.19 ; 1.46)$ & \\
\hline & $\begin{array}{l}\text { Narrative analysis including all } \\
\text { studies }\end{array}$ & $3,746(25)$ & N/A & N/A & \\
\hline \multirow[t]{2}{*}{ Complications } & & $0(0)$ & N/A & N/A & Insufficient evidence \\
\hline & & & & & No studies identified \\
\hline
\end{tabular}

The proportion of patients with seizure freedom was higher in the ERSET trial than the earlier study, where the mean epilepsy duration was longer. However, the patient selection differed between the 2 trials, as ERSET included only patients determined to have surgically remediable mesial temporal lobe epilepsy, ${ }^{2}$ while in the earlier study patients were randomized before the presurgical investigation. ${ }^{1}$

The major strength of the present review is the rigorous protocol for selection and evaluation of the studies. Due to the relatively strict inclusion criteria, studies that might have added a high risk of bias were rejected at the eligibility stage.

We acknowledge that confounding factors might be present in studies favoring shorter epilepsy duration. Patients with epileptogenic lesions and highly congruent preoperative investigations, which are indicators of better seizure outcome, are likely to have surgery earlier than, for instance, patients with normal MRI or complex electroclinical patterns. On the other hand, it is conceivable that patients with higher seizure frequency are selected for presurgical evaluation earlier, which allows for a confounding effect in the opposite direction. The individual studies seldom report the results of preoperative investigations in relation to epilepsy duration. Therefore, we were not able to analyze possible confounding effects arising from different subgroups of patients being selected for surgery earlier. Unknown predictors of seizure outcome may also confound the effect.

The results of the individual meta-analyses are to be interpreted with caution, since populations and surgical procedures vary between studies. Some patients are included in more than one meta-analysis; hence, the meta-analyses should be interpreted as subgroup analyses of different breakpoints.

As shown in the narrative analysis, shorter epilepsy duration is favored in most individual studies, regardless of the population and specific intervention. Several studies include only cases with epileptogenic lesions, which makes early selection of cases with favorable prognosis less likely to explain the results in these studies. Furthermore, the different time intervals studied in the meta-analyses all favor shorter epilepsy duration. We therefore judge that although selection bias may be present in individual studies, the analyses support an association between shorter epilepsy duration and better seizure outcome. The low certainty of evidence acknowledges the inherent weaknesses in the observational study methodology and the risk for residual bias.

Some studies have reported an association between lower age at surgery and a higher proportion of patients with sustained seizure freedom. ${ }^{43,44}$ As younger people obviously have had their epilepsy for a shorter time than older adults, the patient's age at surgery may confound the association between epilepsy duration and seizure outcome. However, a positive association was also found in studies considering both age and epilepsy duration at the time of surgery. ${ }^{18,27-29}$

This review was based on observational studies with moderate risk of bias, which explains the concluding evidence level "low certainty." Randomized studies are usually considered the best evidence base for demonstrating an effect, but for ethical and practical reasons, such studies are not feasible for the targeted question. However, forthcoming methodologically sound observational studies of large, prospectively followed cohorts that 
include strategies to control for confounding factors may strengthen the evidence for this research question. ${ }^{45}$

Finally, we note that few studies report duration of epilepsy after 2 adequate drug trials. Some patients with epilepsy have a long course before drug resistance develops. Based on our study, it is not possible to tell if the duration of drug-resistant epilepsy is a more important predictor than the total epilepsy duration. It is desirable that future studies analyze both variables to clarify this matter.

Duration of epilepsy is the only known modifiable factor that is associated with favorable seizure outcome after epilepsy surgery. This underlines the importance of early referral of patients with drug-resistant epilepsy to improve outcomes. In the meta-analyses, people with shorter epilepsy duration were more likely to be seizure-free at follow-up regardless of the studied cutoff, indicating that earlier surgery is always to be preferred. On the other hand, we found a positive association between shorter duration and seizure freedom also for very long epilepsy durations. This indicates that for patients who are suitable epilepsy surgery candidates, presurgical investigations should be suggested and planned without further delay regardless of epilepsy duration.

\section{Acknowledgment}

The authors thank Sten Anttila, Maja Kärrman Fredriksson, and Nathalie Peira at SBU for their contributions.

\section{Study funding}

The study was financed by Swedish Agency for Health Technology Assessment and Assessment of Social Services, SBU, and grants from the Swedish state under the agreement between the Swedish government and the county councils, the ALF-agreement (grant ALFGBG-723151). The study was not industry-sponsored.

\section{Disclosure}

The authors report no disclosures relevant to the manuscript. Go to Neurology.org/ $\mathrm{N}$ for full disclosures.

\section{Publication history}

Received by Neurology November 17, 2018. Accepted in final form February 21, 2019.

\section{Appendix Authors}

\begin{tabular}{|c|c|c|c|}
\hline Name & Location & Role & Contribution \\
\hline $\begin{array}{l}\text { Johan } \\
\text { Bjellvi, MD }\end{array}$ & $\begin{array}{l}\text { University of } \\
\text { Gothenburg and } \\
\text { Sahlgrenska } \\
\text { University Hospital }\end{array}$ & Author & $\begin{array}{l}\text { Data acquisition, data } \\
\text { analysis and } \\
\text { interpretation, } \\
\text { drafting and revising } \\
\text { the manuscript }\end{array}$ \\
\hline $\begin{array}{l}\text { Ingrid } \\
\text { Olsson, } \\
\text { MD, PhD }\end{array}$ & $\begin{array}{l}\text { University of } \\
\text { Gothenburg }\end{array}$ & Author & $\begin{array}{l}\text { Design and } \\
\text { conceptualization of } \\
\text { study, data acquisition, } \\
\text { data analysis and } \\
\text { interpretation, revising } \\
\text { the manuscript }\end{array}$ \\
\hline
\end{tabular}

Appendix (continued)

\begin{tabular}{|c|c|c|c|}
\hline Name & Location & Role & Contribution \\
\hline $\begin{array}{l}\text { Kristina } \\
\text { Malmgren, } \\
\text { MD, PhD }\end{array}$ & $\begin{array}{l}\text { University of } \\
\text { Gothenburg and } \\
\text { Sahlgrenska } \\
\text { University Hospital }\end{array}$ & Author & $\begin{array}{l}\text { Design and } \\
\text { conceptualization of } \\
\text { study, data acquisition, } \\
\text { data analysis and } \\
\text { interpretation, } \\
\text { drafting and revising } \\
\text { the manuscript }\end{array}$ \\
\hline $\begin{array}{l}\text { Karin Wilbe } \\
\text { Ramsay, } \\
\text { PhD }\end{array}$ & $\begin{array}{l}\text { Swedish Agency for } \\
\text { Health Technology } \\
\text { Assessment and } \\
\text { Assessment of Social } \\
\text { Services, Stockholm }\end{array}$ & Author & $\begin{array}{l}\text { Design and } \\
\text { conceptualization of } \\
\text { study, data acquisition, } \\
\text { data analysis and } \\
\text { interpretation, } \\
\text { drafting and revising } \\
\text { the manuscript }\end{array}$ \\
\hline
\end{tabular}

\section{References}

1. Wiebe S, Blume WT, Girvin JP, Eliasziw M. A randomized, controlled trial of surgery for temporal-lobe epilepsy. N Engl J Med 2001;345:311-318.

2. Engel J Jr, McDermott MP, Wiebe S, et al. Early surgical therapy for drug-resistant temporal lobe epilepsy: a randomized trial. JAMA 2012;307:922-930.

3. Dwivedi R, Ramanujam B, Chandra PS, et al. Surgery for drug-resistant epilepsy in children. N Engl J Med 2017;377:1639-1647.

4. Kwan P, Arzimanoglou A, Berg AT, et al. Definition of drug resistant epilepsy: consensus proposal by the ad hoc Task Force of the ILAE Commission on Therapeutic Strategies. Epilepsia 2010;51:1069-1077.

5. Haneef Z, Stern J, Dewar S, Engel J Jr. Referral pattern for epilepsy surgery after evidence-based recommendations: a retrospective study. Neurology 2010;75:699-704.

6. Rydenhag B, Flink R, Malmgren K. Surgical outcomes in patients with epileptogenic tumours and cavernomas in Sweden: good seizure control but late referrals. J Neurol Neurosurg Psychiatry 2013;84:49-53.

7. Engel J Jr. What can we do for people with drug-resistant epilepsy? The 2016 Wartenberg Lecture. Neurology 2016;87:2483-2489.

8. Erba G, Moja L, Beghi E, Messina P, Pupillo E. Barriers toward epilepsy surgery: a survey among practicing neurologists. Epilepsia 2012;53:35-43.

9. Berg AT, Langfitt J, Shinnar S, et al. How long does it take for partial epilepsy to become intractable? Neurology 2003;60:186-190.

10. Moher D, Liberati A, Tetzlaff J, Altman DG, Group P. Preferred reporting items for systematic reviews and meta-analyses: the PRISMA statement. PLoS Med 2009;6: e1000097.

11. Wieser HG, Blume WT, Fish D, et al. ILAE Commission Report: proposal for a new classification of outcome with respect to epileptic seizures following epilepsy surgery. Epilepsia 2001;42:282-286.

12. Engel J Jr. Outcome with respect to epileptic seizures. In: Engel J Jr, ed. Surgical Treatment of the Epilepsies. New York: Raven Press; 1987:553-571.

13. Guyatt GH, Oxman AD, Vist GE, et al. GRADE: an emerging consensus on rating quality of evidence and strength of recommendations. BMJ 2008;336:924-926.

14. Asadi-Pooya AA, Nei M, Sharan A, Sperling MR. Historical risk factors associated with seizure outcome after surgery for drug-resistant mesial temporal lobe epilepsy. World Neurosurg 2016;89:78-83.

15. Bjørnaes H, Stabell KE, Heminghyt E, Røste GK, Bakke SJ. Resective surgery for intractable focal epilepsy in patients with low IQ: predictors for seizure control and outcome with respect to seizures and neuropsychological and psychosocial functioning. Epilepsia 2004;45:131-139.

16. Dalmagro CL, Bianchin MM, Velasco TR, et al. Clinical features of patients with posterior cortex epilepsies and predictors of surgical outcome. Epilepsia 2005;46: $1442-1449$.

17. Daszkiewicz P, Kowalczyk P, Roszkowski M. Surgical treatment of neuronal-glial tumors of mesial-basal part of temporal lobe: long term outcome and control of epilepsy in pediatric patients. Neurol Neurochir Pol 2018;52:2-8.

18. Edelvik A, Rydenhag B, Olsson I, et al. Long-term outcomes of epilepsy surgery in Sweden: a national prospective and longitudinal study. Neurology 2013;81: 1244-1251.

19. Elsharkawy AE, Pannek H, Schulz R, et al. Outcome of extratemporal epilepsy surgery experience of a single center. Neurosurgery 2008;63:516-6.

20. Fauser S, Bast T, Altenmüller DM, et al. Factors influencing surgical outcome in patients with focal cortical dysplasia. J Neurol Neurosurg Psychiatry 2008;79:103-105.

21. Hennessy MJ, Elwes RD, Honavar M, Rabe-Hesketh S, Binnie CD, Polkey CE. Predictors of outcome and pathological considerations in the surgical treatment of intractable epilepsy associated with temporal lobe lesions. J Neurol Neurosurg Psychiatry 2001;70:450-458.

22. Janszky J, Janszky I, Schulz R, et al. Temporal lobe epilepsy with hippocampal sclerosis: predictors for long-term surgical outcome. Brain 2005;128:395-404.

23. Kanner AM, Byrne R, Chicharro A, Wuu J, Frey M. A lifetime psychiatric history predicts a worse seizure outcome following temporal lobectomy. Neurology 2009;72: 793-799. 
24. Liava A, Mai R, Cardinale F, et al. Epilepsy surgery in the posterior part of the brain. Epilepsy Behav 2016;64:273-282.

25. Luyken C, Blümcke I, Fimmers R, et al. The spectrum of long-term epilepsyassociated tumors: long-term seizure and tumor outcome and neurosurgical aspects. Epilepsia 2003;44:822-830.

26. McIntosh AM, Kalnins RM, Mitchell LA, Fabinyi GC, Briellmann RS, Berkovic SF. Temporal lobectomy: long-term seizure outcome, late recurrence and risks for seizure recurrence. Brain 2004; 127:2018-2030.

27. Meguins LC, Adry RA, Silva-Junior SC, Araújo Filho GM, Marques LH. Shorter epilepsy duration is associated with better seizure outcome in temporal lobe epilepsy surgery. Arq Neuropsiquiatr 2015;73:212-217.

28. Patra S, Elisevich K, Podell K, et al. Influence of age and location of ictal onset on postoperative outcome in patients with localization-related epilepsy. $\mathrm{Br} \mathrm{J}$ Neurosurg 2014;28:61-67.

29. Pelliccia V, Deleo F, Gozzo F, et al. Early and late epilepsy surgery in focal epilepsies associated with long-term epilepsy-associated tumors. J Neurosurg 2017;127:1147-1152.

30. Radhakrishnan A, Abraham M, Vilanilam G, et al. Surgery for long-term epilepsy associated tumors (LEATs): seizure outcome and its predictors. Clin Neurol Neurosurg 2016;141:98-105.

31. Ramantani G, Stathi A, Brandt A, et al. Posterior cortex epilepsy surgery in childhood and adolescence: predictors of long-term seizure outcome. Epilepsia 2017;58:412-419.

32. Ramantani G, Kadish NE, Mayer H, et al. Frontal lobe epilepsy surgery in childhood and adolescence: predictors of long-term seizure freedom, overall cognitive and adaptive functioning. Neurosurgery 2018;83:93-103.

33. Salanova V, Markand O, Worth R. Temporal lobe epilepsy surgery: outcome, complications, and late mortality rate in 215 patients. Epilepsia 2002;43:170-174.

34. Schramm J, Kuczaty S, Sassen R, Elger CE, von Lehe M. Pediatric functional hemispherectomy: outcome in 92 patients. Acta Neurochir 2012;154:2017-2028.
35. Simasathien T, Vadera S, Najm I, Gupta A, Bingaman W, Jehi L. Improved outcomes with earlier surgery for intractable frontal lobe epilepsy. Ann Neurol 2013;73: 646-654.

36. Stavem K, Bjørnaes H, Langmoen IA. Predictors of seizure outcome after temporal lobectomy for intractable epilepsy. Acta Neurol Scand 2004;109:244-249.

37. Sun Z, Zuo H, Yuan D, et al. Predictors of prognosis in patients with temporal lobe epilepsy after anterior temporal lobectomy. Exp Ther Med 2015;10:1896-1902.

38. Swarztrauber K, Dewar S, Engel J. Patient attitudes about treatments for intractable epilepsy. Epilepsy Behav 2003;4:19-25.

39. de Flon P, Kumlien E, Reuterwall C, Mattsson P. Empirical evidence of un derutilization of referrals for epilepsy surgery evaluation. Eur J Neurol 2010;17: 619-625.

40. Prus N, Grant AC. Patient beliefs about epilepsy and brain surgery in a multicultural urban population. Epilepsy Behav 2010;17:46-49.

41. Erba G, Messina P, Pupillo E, Beghi E, Group O. Acceptance of epilepsy surgery among adults with epilepsy: what do patients think? Epilepsy Behav 2012;24: 352-358.

42. Hrazdil C, Roberts JI, Wiebe S, et al. Patient perceptions and barriers to epilepsy surgery: evaluation in a large health region. Epilepsy Behav 2013;28:52-65.

43. de Tisi J, Bell GS, Peacock JL, et al. The long-term outcome of adult epilepsy surgery, patterns of seizure remission, and relapse: a cohort study. Lancet 2011;378 1388-1395.

44. Schwartz TH, Jeha L, Tanner A, Bingaman W, Sperling MR. Late seizures in patients initially seizure free after epilepsy surgery. Epilepsia 2006;47:567-573.

45. Beghi E. Methodological demands on observational studies of outcomes after epilepsy surgery. In: Malmgren K, Baxendale SA, Cross H, eds. Long-term Outcomes of Epilepsy Surgery in Adults and Children. Heidelberg: Springer; 2015 $5-18$. 


\section{Neurology}

\section{Epilepsy duration and seizure outcome in epilepsy surgery: A systematic review and meta-analysis}

Johan Bjellvi, Ingrid Olsson, Kristina Malmgren, et al.

Neurology 2019;93;e159-e166 Published Online before print June 10, 2019

DOI 10.1212/WNL.0000000000007753

This information is current as of June 10, 2019

\section{Updated Information \&} Services

References

Citations

Subspecialty Collections

Permissions \& Licensing

Reprints including high resolution figures, can be found at: http://n.neurology.org/content/93/2/e159.full

This article cites 43 articles, 9 of which you can access for free at: http://n.neurology.org/content/93/2/e159.full\#ref-list-1

This article has been cited by 7 HighWire-hosted articles: http://n.neurology.org/content/93/2/e159.full\#\#otherarticles

This article, along with others on similar topics, appears in the following collection(s):

All Epilepsy/Seizures

http://n.neurology.org/cgi/collection/all_epilepsy_seizures

Clinical trials Systematic review/meta analysis

http://n.neurology.org/cgi/collection/clinical_trials_systematic_review_ meta_analysis_

Epilepsy surgery

http://n.neurology.org/cgi/collection/epilepsy_surgery_

Information about reproducing this article in parts (figures,tables) or in its entirety can be found online at:

http://www.neurology.org/about/about_the_journal\#permissions

Information about ordering reprints can be found online:

http://n.neurology.org/subscribers/advertise

Neurology ${ }^{\circledR}$ is the official journal of the American Academy of Neurology. Published continuously since 1951, it is now a weekly with 48 issues per year. Copyright Copyright (C) 2019 The Author(s). Published by Wolters Kluwer Health, Inc. on behalf of the American Academy of Neurology.. All rights reserved. Print ISSN: 0028-3878. Online ISSN: 1526-632X.

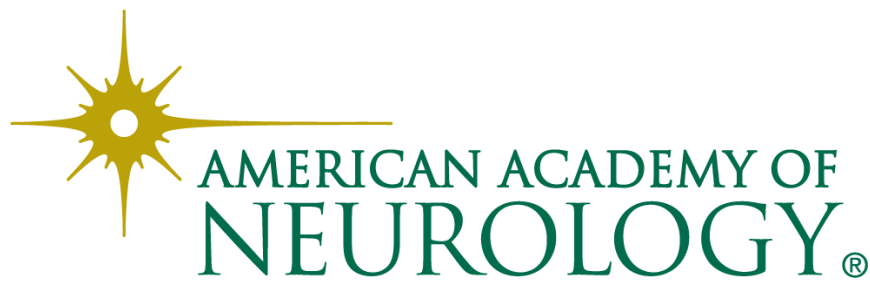

